\title{
JOINT RANGE SYSTEMS INTEROPERABILITY ACHIEVED THROUGH THE IMPLEMENTATION OF THE TEST AND TRAINING ENABLING ARCHITECTURE (TENA)
}

\begin{abstract}
The Foundation Initiative 2010 (FI 2010) project, sponsored by the Office of the Secretary of Defense (OSD) Central Test and Evaluation Investment Program (CTEIP), has developed and is continuing to refine a common architecture and requisite software used to integrate testing, training, and simulation systems distributed across many DoD test and training range facilities. The Test and Training Enabling Architecture (TENA), has been successfully implemented on $\mathrm{DoD}$ and commercial range instrumentation systems, used as a reusable enabler of distributed, live United States Joint Forces Command (USJFCOM) and Joint National Training Capability (JNTC) exercises.
\end{abstract}

\section{B. Gene Hudgins (Primary) \\ TENA Development Team Lead \\ 557 Mary Esther Cut-Off \\ Fort Walton Beach, FL 32548}

Jason Lucas

FI 2010 Deputy Technical Project Manager

201 West Eglin Blvd, Suite 258

Eglin AFB FL 32542

\section{KEYWORDS}

Range Systems Interoperability, Resource Resuse, TENA, Middleware 


\section{INTRODUCTION}

Just over a year ago, miltary events in Iraq, a country the size of California and with a population of about 25 million, transfixed the population of United States and, indeed, the world. Iraq was feeling the prosecution of Operation Iraqi Freedom (OIF) by the United States Military and its coalition partners.. As Iraq's dust-blown landscape was being crisscrossed at a lightning-fast pace by U.S. forces with the ancient city of Baghdad at the converging point, the day and night skies over Iraq were filled with swarms of aircraft and missiles, each using the ultimate in target identification and guidance systems. This rapid domination aspect, the "Shock and Awe," of the operation would last just over three weeks and would bring all organized Iraqi military forces to their knees. While the operation's success can be largely found in its Joint Forces application of military power, it is also found in the military's devotion to "training the way you fight." The result of that fieldproven approach can be read in the Third Infantry Division (Mechanized) After Action Report, Operation Iraqi Freedom (3 July 2003) (http://www.globalsecurity.org/military/library/report/2003/3id-aar-jul03.pdf): "The roots of the division's successful attack to Baghdad are found on the training fields of Fort Steward, Fort Irwin, and Kuwait. The division crossed the line of departure with a mature and trained group of staff officers, commanders, and soldiers." But it is not enough to gather military forces in a difficult environment and subject them to individual and group challenges, the measure of the training of the men and women of the military and the weapon systems they use must be taken accurately and timely.

On the geographically dispersed Department of Defense (DoD) land, air, and sea test and training ranges that dot the United States from border to border and ocean to ocean is where much of the warfighter's training takes place. These ranges have prepared the U.S. warfighters for many years. Some early ranges, using wooden rifles, cut-out tanks, and airplanes dropping sacks of flour, trained troops before World War II. But now, the complexity of weapons and the complexity of weapons training have increased tremendously. And as the complexity of systems and system events has grown, so have the number of data points that give value to the event and the equipment required to capture those data points. This technology and sophisticated growth of weapons, weapon systems, and systems of systems has spiraled upward at an ever-increasing rate, particularly in the last three decades, driving a parallel evolution of the range instrumentation systems required to capture and translate the data generated on the range. This data, streaming in to collection points from sensors and telemetry systems, provides weapon and training systems affirmation, or, perhaps more importantly, can ensure safe and effective weapon system operation and training. The data also invariably affects almost every aspect of range operation and management, from mission definition to budget approval. 
As each range's mission was evolving and expanding in response to the technology of weapons growth, the evolving instrumentation suites, including the sensors, hardware, software, and networks, grew in a vertical "stovepipe" fashion that was often individual to the range. Individual ranges became effective within their boundaries; however, their ability to share data laterally in real-time was minimal. This vertical growth did not allow for taking easy advantge of the growth in modeling and simulation and its revolutionary application to training. It also did not allow easy movement to range resource reuse and range interoperability, concepts that were being forwarded in the late 1990s by the Foundation Initiative 2010 (FI 2010) project, which is sponsored by the Office of the Secretary of Defense (OSD) Central Test and Evaluation Investment Program (CTEIP). To bring the efficiency and economic advantages of interoperability and reuse to the DoD test and training ranges, FI 2010 has developed and is continuing to refine its Test and Training Enabling Architecture, TENA.

Interoperability can be defined as the characteristic of an independently-developed software element that enables it to work together with other elements toward a common goal. Interoperability focuses on what is common among software elements. Reuse is the ability to use a software element in a context for which it was not originally designed, so reuse focuses on the multiple uses of a single element, and it requires well documented interfaces. To achieve interoperability, a common architecture, an ability to meaningfully communicate (including a common language and a common communication mechanism), and a common context (including the environment and time) must be present.

\section{DOD INITIATIVES LEAD TO INTEROPERABILITY, REUSE AND TENA}

TENA was not the initial concept at achieving interoperability and reuse; this enabling architecture and requisite software came from an evolutionary track that began with two DoD initiatives, the Joint Advanced Distributed Simulation (JADS) Program and the High Level Architecture (HLA) for Modeling and Simulation (M\&S). The JADS program was a joint experiment to determine the feasibility of using simulators in testing environments, to investigate new evaluation methodologies, and to determine economic and performance benefits of integrated simulator technologies. JADS confirmed the usefulness of combining simulators with live systems for test and evaluation (T\&E) purposes. HLA, sponsored by the Defense Modeling and Simulation Office (DMSO), created a technical architecture for the DoD modeling and simulation community to promote interoperability and reuse among simulation assets. TENA builds on the efforts pioneered in JADS and the HLA to support interoperability between the live testing/training range domain and the larger modeling and simulation community.

Since achieving true range interoperability requires the use of a common architecture (including a common language, communication mechanism and context [including the 
environment and time]) to meaningfully communicate across divergent systems, and range resource reuse requires well-documented system interfaces that ensure commonality, the TENA architecture is a technical blueprint for achieving that vision of an interoperable, composable set (composibility is defined as the ability to rapidly assemble, initialize, test, and execute a system from members of a pool of reusable, interoperable elements) of geographically distributed range resources-some live, some simulated-that can be rapidly combined to meet new testing and training missions in a realistic manner.

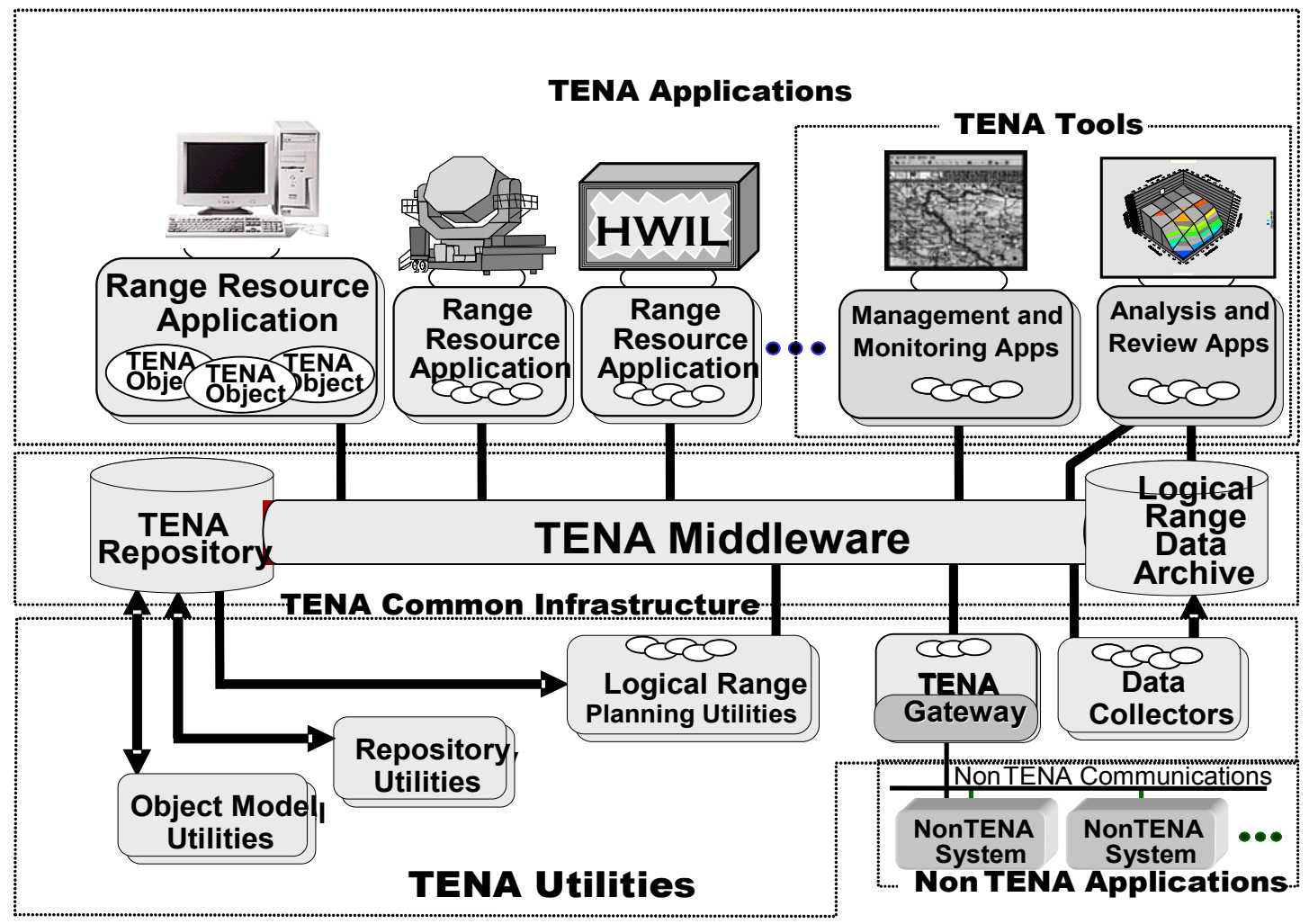

Figure 1. TENA Architecture Overview

Please refer to Figure 1. TENA includes several components, including a domain-specific object model that supports information transfer throughout the event lifecycle, common real-time and non-real-time software infrastructures for manipulating objects; as well as standards, protocols, rules, supporting software, and other key components. Within the TENA concept, a common Logical Range Object Model (LROM) is defined as that which is linked into all interoperating applications on the network. This object model defines the TENA objects that are available for any participating application to either publish (send), subscribe (receive), or both. The definition of the object model and the use of common middleware data distribution enable interoperability. Each interoperating application may translate from the TENA object model formats to local data representations as required. 
Through the use of utilities and a growing number of common tools, TENA also provides enhanced capability to accomplish the tasks which have been routinely performed on the test and training ranges in support of exercises. Refer again to Figure 1. The steps in many of the tasks are automated and information flow is streamlined between tools and the common infrastructure components through the enhanced software interoperability provided by TENA. TENA utilities facilitate the creation of TENA-compliant software and installing, integrating, and testing the software at each designated range. This complex task falls to the Logical Range Developer, which, in this phase, performs the detailed activities described in the requirement definitions and event planning, and the event construction, setup, and rehearsal activities of the Logical Range Concept of Operations. While there is still a lot of manual exercise and event setup required at ranges, TENA tools, as they are developed and become accepted across the range community, will make exercise pre-event management easier.

\section{MILITARY EXERCISES USE TENA}

TENA has become an important factor in range instrumentaion and data gathering systems, particularly as an interoperability enabler in United States Joint Forces Command (USJFCOM) Joint National Training Capability (JNTC) military exercises. In March of this year, Major General Gordon C. Nash, United States Marine Corps Commander, Joint Warfighting Center and Director for Joint Training, USJFCOM, spoke before the House Armed Services Subcommittees on Readiness, Terrorism, Unconventional Threats, and Capabilities on the JNTC. Addressing Range Instrumentation as part of the progress in implementing the JNTC, Maj. Gen. Nash said that JNTC was playing a key role in upgrading instrumentation systems employed on the many service ranges used for test and training throughout the country. "These upgrades, employing a consistent set of standards and protocols, are ensuring a level of service interoperability never seen before. Additionally, through the investment incentive offered by the JNTC Joint Management Office, modernization of service-centric range instrumentation and telemetry systems is moving forward at an accelerated pace. Modern instrumentation systems will comply with the Test and Training Enabling Architecture (TENA), an architecture and interoperability standard that shares information among instrumentation systems, simulations, and realworld command-and-control systems." Further in his speech, Maj. Gen. Nash addressed the use of web-based technologies to investigate and develop advanced concepts in joint training. One example was that "a web-based repository browser is being developed to hold all object model specification requirements for the Test and Training Enabling Architecture (TENA). This repository acts as the access mechanism to build an instance of a TENA event." http://www.jfcom.mil/newslink/storyarchive/2004/sp031804.htm). (pages 18,20 of 24) (See figure 1 in this paper for representation of the repository). 
As for joint training successes, while there were earlier ones, Maj. Gen. Nash said a "bigger opportunity" occurred in January 2004 when the JNTC conducted a large-scale military exercise, the Western Range Complex (WRC) Horizontal Training Event. Designed to test and evaluate warfighters and warfighting concepts, the exercise spread its personnel and equipment and its data gathering and distribution networks from coast to coast, from the Fort Irwin and Twentynine Palms ranges in California to the USJFCOM location in Suffolk, Virginia, with several exercise and data points scattered between the two coasts in Nevada, New Mexico, Kansas, Alabama, Florida, and Georgia. Hundreds of air sorties and days of ground maneuvering played out the various scenarios as exercise data flowed from several disparate instrumentation systems: Advanced Range Data Systems (ARDS) for fixed and rotary wing aircraft, ARDS-Lite (a scaled-down ARDS) for ground vehicles, Integrated GPS Radio System (IGRS) for ground troops and vehicles, and Air Warrior for fixed wing aircraft. TENA was designated by JNTC to "connect" these individually distinct systems and the geographically separated ranges and allow a smooth data flow to TENA-enabled displays and data analysis points.

The WRC exercise was the first pre-initial operating capability, Live, Virtual, and Constructive (LVC) event conducted by the JNTC. The event's live activities were conducted on the United States Western Ranges in conjunction with a U. S. Army National Training Center (NTC) rotation, a U.S. Air Force Air Warrior segment, and a U. S. Marine Corps Combined Arms eXercise (CAX) at Marine Corps Station Twentynine Palms, California. Devised for the exercise, the integration and instrumentation solution (See Figure 2) linked the exercise locations using TENA. Live air and ground tracks from the NTC, Nellis AFB (Air Warrior), and Twentynine Palms ranges were integrated, and then distributed to USJFCOM to create a LVC Joint training environment. Resource reuse, a TENA capability, was demonstrated with the utilization of the RangeView and the Personal Computer Debriefing System (PCDS) as data displays in the exercise. TENA implementations of these systems were developed for previous events. The data from January's Horizontal Event indicates that TENA performance met the JNTC expectations, and it will be used for the remainder of the JNTC 2004 exercises. However, striving to improve and refine the middleware and its uses, TENA users and developers gathered "lessons learned" during and following the January event. For example, (1) continue to refine the JNTC Object Model (JOM) and retrofit the changes to applications already used in JNTC events in order to build toward a set of "plug and play" applications that can quickly be drawn from to support future Live JNTC events; (2) define and implement comprehensive JNTC event Participant Identification and Time-History and Event Archive Tools; and (3) improve coordination between the JNTC event Range Integration, JNTC communications and networking support team, analysis team and scenario/force structure activities. It is expected these and other lessons learned will improve TENA's overall performance. 


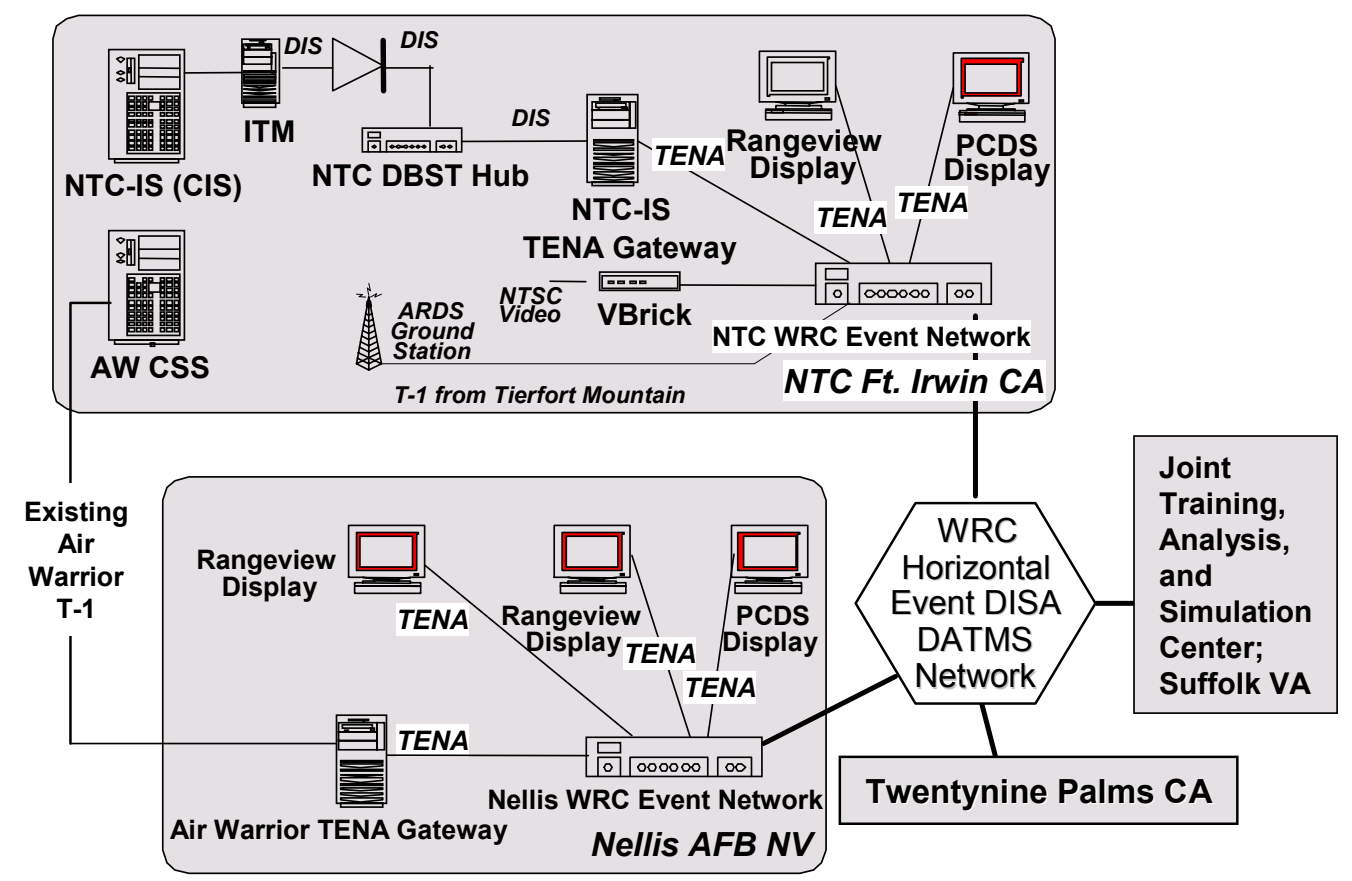

Figure 2. Integration and Instrumentation Solution

However, TENA's first use in a military exercise was in Millennium Challenge 2002 (MC02). MC02, a USJFCOM exercise held in July and August 2002, was a joint experiment, transformation event, and major field exercise. The exercise was designed to test some of the then current key USJFCOM strategic concepts and capabilities and to concentrate on the future concepts and capabilities of the U.S. Army, U.S. Navy, U.S. Marine Corps, U.S. Air Force, and the Special Operations Command. This also extended to the data-gathering and data-distribution networks used throughout the range community for testing and training, and in particular, the concepts and capabilities of range interoperability and resource reuse. For the MC02 live events, TENA was chosen as the data distribution system enabler.

USJFCOM and a MC02 Western Range consortium developed and designed a range integration package which supported the then USJFCOM Commander's intent that "...the live portion of the event will...demonstrate the ability to link existing ranges within a joint event...". This goal was accomplished by extracting live-force participants' track and status data at the instrumented data fusion points at each range and delivering the data to TENA software gateways which "standardized" the various range data formats. The standardized data was then distributed over the MC02 Range Integration network to the USJFCOM Joint Training, Analysis, and Simulations Center and other event locations, where the live data was fused with the model federation picture. Also included in the original intent for MC02 was the need to provide a seamless "ground truth" picture of the 
combined live and virtual joint forces participating in the event. Accomplishment of the MC02 goals depended largely on a data distribution system capable of seamlessly linking the various data points, requirements that even at that early stage of TENA development were well within the range of TENA capabilities.

The overall concept of the MC02 Range Integration effort was to share time-space-position information (TSPI) data between the ranges involved in the event. This data were distributed in a common format and represented on a common range display such that it was transparent to the casual viewer that the data was coming from several different sites across the country. Any editing/smoothing was left as a range-specific function in an effort to remain as close as possible to "range ground truth". As shown in figure 3, the scope of this Range Integration effort was to integrate TSPI data from the following range facilities: Land Range (LR) and Electronic Combat Range (ECR) at NAVAIR, China Lake, California; Sea Range (SR) at NAVAIR, Pt. Mugu, California; National Training Center (NTC) at Fort Irwin, California; Nellis AFB, Nevada; Southern California Logistics Airport (SCLA), and Southern California Offshore Range (SCORE) with the simulated environment of the MC02 project. Each range had the capability to send, receive, display, filter, and log the data using the gateway approach with a custom user interface and a common display picture.

The initial concept of the gateway implementation was to build a core piece of software with as many common features as possible based on the TENA Middleware framework using a common MC02 object model. Two legacy range applications were adapted to use the TENA Middleware for MC02. These two applications, the TSPI Internal Entity Reformatter (TIER) and the RangeView display application, provided range system interfaces, local data format translations, and display capabilities. TIER was adapted to use TENA as a "Gateway," translating range information from the legacy application format to the TENA objects, while RangeView was made a native TENA display application that would be used in future exercises. TIER implemented the unique range data systems interfaces, and RangeView provided additional display and analysis capabilities.

As the conduit for Ground Truth TSPI data from the various ranges to Command and Control Systems at USJFCOM, TENA met the test objectives for each of the exercise rehearsals and, during the $\mathrm{MC} 02$ execution, worked reliably for days without failure.

TENA also offers a significant benefit, realized in Millennium Challenge 2002, auto-code generation. The TENA Middleware is designed to enable the rapid development of distributed applications that exchange data using the publish-subscribe paradigm. While many publish-subscribe systems exist, few possess the high-level programming abstractions presented by the TENA Middleware. The TENA Middleware provides these high-level abstractions by using auto-code generation to create a complex Common Object Request Broker Architecture (CORBA) application. As such, the TENA Middleware offers 
programming abstractions not present in CORBA and provides a strongly-type-checked framework interface that is much less error-prone than the existing CORBA API. These higher-level programming abstractions combined with a framework designed to reduce programming errors enable users to quickly and correctly express the concepts of their applications. Re-usable standardized object interfaces and implementations further simplify the application development process.

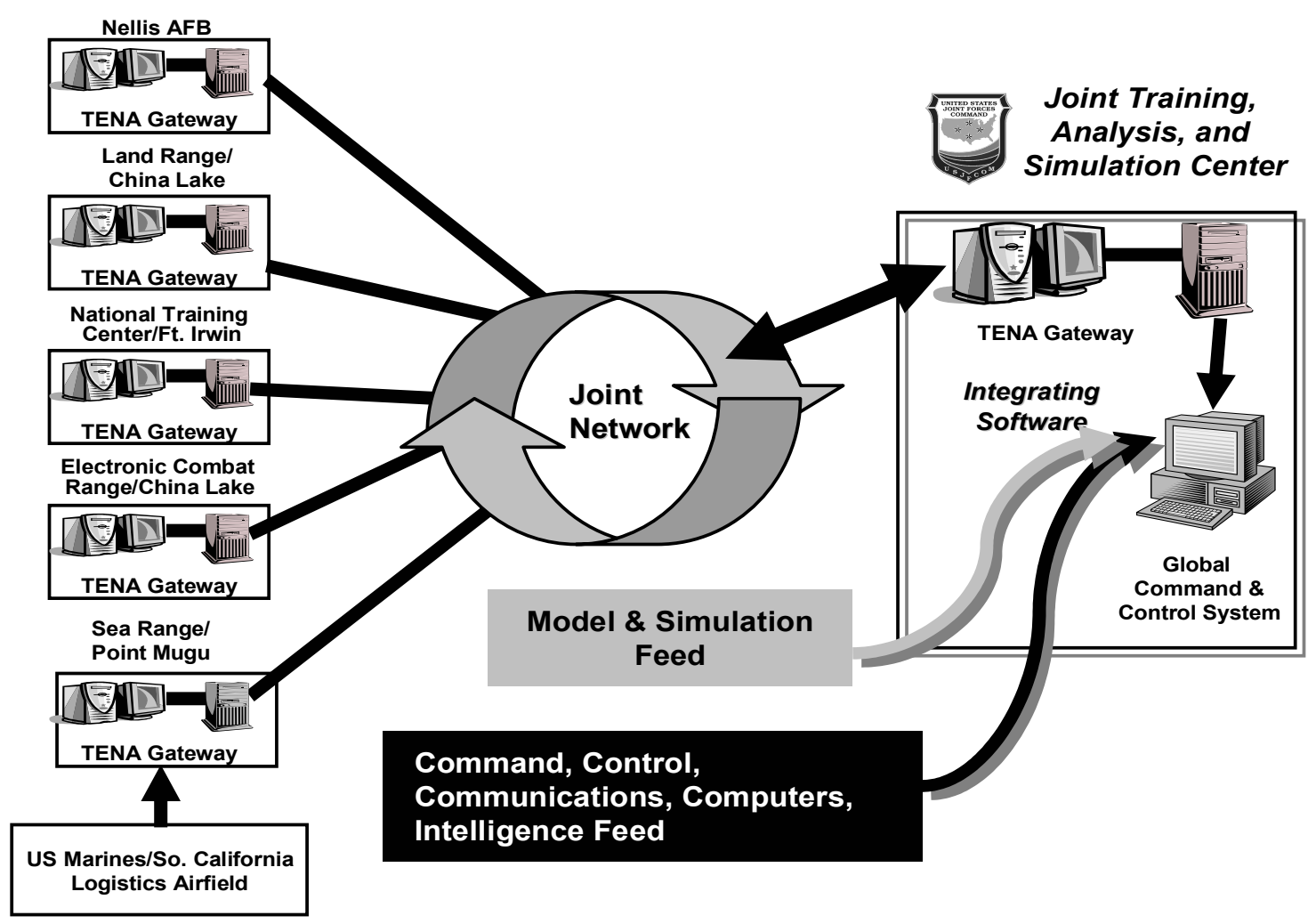

Figure 3. MC02-TENA Range Integration

TENA's performance in Millennium Challenge 2002 met all requirements, and it was accepted as the JNTC Instrumentation Architecture and scheduled for use in the USJFCOM exercise, Roving Sands 2003. However, as the exercise was entering the final planning stages, Operation Iraqi Freedom drew most of the scheduled participants to a real conflict thousands of miles away, and Roving Sands 2003 became a mostly simulated exercise.

To replace the live-event portion of Roving Sands 2003, the Joint Combat Identification Evaluation Team (JCIET), a multi-Service unit at Eglin Air Force Base, Florida, whose mission is to reduce battlefield fratricide, collaborated with USJFCOM to schedule a liveevent exercise, Joint Combat Identification Exercise 2003 (Joint CIDEX 2003), for August 2003. Working through the short planning cycle, JNTC Range Integration and 
Instrumentation and members of the FI 2010 TENA Development Team made TENA a parallel part of the data network, allowing an evaluation of the middleware's interoperability capability.

The Joint CIDEX 2003 scenario was staged across four southern states and the northern Gulf of Mexico as a small ground war in southern Mississippi supported by air-to-ground and air-to-air missions flown by fixed-wing aircraft instrumented with ARDS pods. The scenario also employed Former Soviet Union (FSU) air defense vehicles, CH-47 Chinook and Mi-8 Hip helicopters, and Navy RQ1L Predator Unmanned Aerial Vehicles (UAVs), all of which were equipped with ARDS-Lite. Fort Rucker, Alabama, provided a Virtual Constructive Simulation capability for the exercise. TENA's parallel role to the datagathering systems was that of an interoperability interface between the employed individually distinct instrumentation systems in the aircraft and ground systems. A TENA object model, which had been originally developed for Roving Sands 2003, and RangeView, the native TENA data display developed for MC02, were used in the exercise, demonstrating the resource reuse capability of TENA.

\section{CONCLUSION}

In his book, Wars of the Jews (78 A.D), the Jewish historian Flavius Josephus observed and wrote about Roman soldiers as they prepared almost daily for battle, saying their "exercises were unbloody battles, and their battles bloody exercises. It is the reason they bear the fatigue of battle so easily." Today's military exercises range from small-unit maneuvering to large-scale Joint Services exercises, such as Millennium Challenge and the Joint National Training Capability exercises scheduled for 2004. Simulated and constructive and live-fire events are blended to present representative scenarios spread across several ranges. The data collected, whether from a maneuvering tank platoon, or the employment

of armor and infantry divisions and hundreds of aircraft sorties, is invaluable in preparing the warfighters to "bear the fatigue of battle." As the DoD land, sea, and air test and training ranges embrace the challenge of supporting the warfighters training needs, range interoperability and range resource reuse become increasingly important. FI 2010's TENA Middleware offers effective, efficient, and economical solutions to those requirements. Additional information about TENA can be found at its website: http://www.fi2010.org. 